\title{
Efeitos da descentralização da saúde básica no Brasil sobre o nível de corrupção em municípios investigados pelo governo central
}

Effects of primary health decentralization in Brazil on the corruption level of municipalities investigated by the central government

\begin{abstract}
The aim of this paper is to study the relationship between decentralization and corruption in Brazil during the 2007-2010 period using corruption information from the anti-corruption program by the Comptroller General of the Union. We investigate whether the results of Peixoto et al. (2012) for the Southeast region are valid for the country as a whole. These estimates are important because Brazil has a very unequal regional socioeconomic pattern and because the decentralization process is still ongoing. Two measures of decentralization were used: an indicator of fiscal decentralization and a measure of administrative decentralization. Results suggest that there is a negative and statistically significant relationship between administrative decentralization and corruption in health programs managed by municipalities, in contrast to the previous evidence for the Souhteast region. On the other hand, no significant change is observed on the effects of financial decentralization.
\end{abstract}

\section{Keywords}

decentralization; corruption; health sector; counting data.

JEL Codes H51; D73.

\author{
Marislei Nishijima \\ Universidade de São Paulo \\ Fernando Antonio Slaibe Postali \\ Universidade de São Paulo \\ Fabiana Fontes Rocha \\ Universidade de São Paulo
}

\section{Resumo}

O objetivo deste artigo é estudar a relação entre descentralização e corrupção no Brasil entre 2007 e 2010, usando como medida de corrupção as irregularidades descritas nos relatórios das auditorias do programa de fiscalização dos municípios da Controladoria Geral da União. O artigo procura verificar se os resultados encontrados para a região Sudeste no periodo de 2004 a 2006 (Peixoto et al., 2012) podem ser generalizados. Considerando a imensa diferença regional do país, a simples inferência a partir de resultados da região mais desenvolvida e rica apresenta riscos. Além disso, o processo de descentralização ainda está acontecendo. Usamos como indicadores de descentralização uma variável de descentralização financeira e outra de descentralização administrativa. Os resultados sugerem que a descentralização administrativa, diferentemente do resultado obtido para a região Sudeste em periodo anterior, reduz o número de ocorrências caracterizadas como corrupção junto aos programas de saúde geridos pelos municípios auditados. Entretanto, nenhum efeito da descentralização financeira se mostrou significativo.

\section{Palavras-chave}

descentralização; corrupção; setor saúde; dados de contagem.

Códigos JEL H51; D73. 


\section{Introdução}

O processo de universalização e descentralização da saúde no Brasil tem início com a Constituição de 1988, quando foi criado o Sistema Único de Saúde (SUS). O SUS se organizou a partir de uma estrutura hierárquica em que os municípios são encarregados dos cuidados básicos em saúde, enquanto os procedimentos mais complexos são de responsabilidade dos governos estaduais e dos grandes municípios. O governo federal é responsável pelas diretrizes da política de saúde e pela transferência dos recursos necessários para que os estados e os municípios executem as ações de saúde que lhe foram atribuídas. Embora a responsabilidade pelo financiamento tenha sido definida também de maneira compartilhada, apenas a participação do governo federal foi inicialmente inserida nas disposições constitucionais transitórias.

No início de 1990, uma crise de financiamento se estabeleceu no setor brasileiro de saúde, cuja origem pode ser atribuída tanto à má definição das responsabilidades de cada esfera de governo na provisão de saúde, quanto à falta de clareza sobre as fontes de financiamento e os níveis de comprometimento de cada esfera de governo nas despesas do setor. $\mathrm{Na}$ verdade, o financiamento da saúde no Brasil é marcado por um debate em que muitos acreditam que a promessa de um sistema universal de saúde só pode ser cumprida com aumento do gasto público. Embora a parcela do gasto do governo em saúde com relação ao gasto total do governo tenha aumentado ao longo do tempo, passando de 4,1\% em 2000 para 6,9\% em 2013, esse montante é baixo comparado ao observado em outros países. Na França, no Reino Unido e nos Estados Unidos, os gastos públicos com saúde correspondiam a 15,8\%, 13\% e 16\% do gasto público total em 2013, respectivamente (World Health Organization, 2013).

Fato é que estados e municípios relutaram em destinar recursos próprios ao financiamento da saúde, procurando contar com as transferências federais para isso. Foi somente com a Emenda Constitucional 29/2000, com a vinculação dos recursos das três esferas de governo para o financiamento das ações de saúde, que de fato o processo de descentralização definido na Constituição começou a tomar forma.

Apesar da orientação pró-descentralização, do ponto de vista teórico não há consenso na literatura de que a transferência de responsabilidade de gasto (arrecadação) para estados/municípios é com certeza benéfica. $\bigcirc$ 
principal argumento a favor da descentralização segue a racionalidade inicialmente expressa por Tiebout (1956), segundo a qual a provisão de bens públicos a nível local leva à maior variedade e à maior adequação no atendimento das preferências das populações locais. A descentralização traria, assim, uma melhora na eficiência alocativa. Existem, no entanto, uma série de imperfeições na provisão de serviços a nível local que podem impedir que os benefícios da descentralização sejam observados (Tanzi, 2000).

Uma dessas imperfeições consiste na existência de instituições locais menos desenvolvidas do que instituições nacionais, o que acaba fazendo com que haja uma relação positiva entre descentralização e corrupção. ${ }^{1}$

No entanto, este também é um ponto de disputa. Modelos de concorrência interjurisdicional, como o de Brennan e Buchanan (1980), apontam para uma relação negativa entre descentralização e corrupção decorrente da concorrência entre governos locais que atrairia residentes e assim limitaria o poder dos burocratas em extrair renda pela prestação de serviços. Careaga e Weingast (2000), Rodden (2000) e Fan, Lin e Treisman (2009), por outro lado, observam que a descentralização de gastos, sem a correspondente descentralização de receitas não conduz, necessariamente, à menor corrupção, uma vez que as transferências fiscais levam os governantes locais a relaxar o esforço fiscal. Persson e Tabellini (2000) constroem um modelo em que os agentes numa burocracia centralizada são responsáveis por várias tarefas que afetam vários municípios, enquanto numa burocracia descentralizada são responsáveis por uma tarefa específica numa única jurisdição. Na burocracia descentralizada o agente é, portanto, o responsável direto pelos seus atos, o que leva a uma accountability mais direta e a uma melhora no desempenho, o que em última instância levaria a uma associação negativa entre descentralização e corrupção.

A literatura empírica sobre o assunto é bastante escassa. Fisman e Gatti (2002a), usando uma amostra de 57 países para o período 1980-1995, encontram uma associação negativa entre descentralização e corrupção ${ }^{2}$. Fisman e Gatti (2002b) avaliam se o descompasso entre a descentralização de receitas e despesas conduz à maior corrupção, usando dados para os estados americanos. Concluem que a corrupção é maior nos estados

1 Brueckner (1999), por exemplo, aponta que corrupção é, provavelmente, um problema entre os governos locais.

$2 \mathrm{Na}$ verdade eles estimam uma regressão cross-country, uma vez que trabalham com a média das variáveis. 
que recebem maiores níveis de transferências federais. Mais recentemente, Fredriksson e Vollebergh (2009), avaliando os países da OECD, obtêm evidência indireta de que em sistemas federativos a corrupção é menor porque o custo da corrupção é maior, dado que um número muito maior de burocratas deve ser induzido com propinas.

Goel e Nelson (2011) investigam os efeitos da descentralização sobre a corrupção nos Estados Unidos, levando em consideração os efeitos para além do aspecto da descentralização financeira, conforme o escopo de serviços ofertados e a população atendida. Seus resultados apontam para efeitos diferentes da descentralização conforme o tipo. Descentralizações com propósitos mais gerais estão positivamente correlacionadas com corrupção, enquanto as com finalidades mais específicas estão negativamente correlacionadas.

O objetivo deste artigo é verificar a associação entre descentralização na área de saúde e corrupção, sendo a medida de corrupção obtida a partir dos relatórios dos auditores que fiscalizam os programas federais executados de forma descentralizada pelos municípios no âmbito do Programa de Fiscalização de Município Pequenos e Médios, por meio de Sorteios Públicos da Controladoria Geral da União (CGU). ${ }^{3}$ Com base nos relatórios mencionados, foram contabilizadas as irregularidades detectadas em três programas: o Atendimento Assistencial nos Municípios Brasileiros (também descrito com PAB-Fixo), o Programa Saúde da Família e a Estruturação da Rede de Serviços de Atenção Básica da Saúde. Esses programas foram escolhidos por constituírem parte integrante fundamental da estratégia de atenção básica de saúde.

Além desta introdução, o artigo está organizado da seguinte maneira: a segunda seção apresenta os materiais e métodos de construção das variáveis, as informações sobre irregularidades nos diferentes programas de atenção básica de saúde e discute quais delas podem ser consideradas corrupção. Também lista brevemente as diferentes medidas de descentralização existentes na literatura e discute as dificuldades envolvidas na mensuração da autonomia dos governos subnacionais. A terceira seção apresenta a estratégia metodológica envolvendo métodos e teoria. A quarta seção apresenta os resultados e uma breve discussão. Finalmente, a quinta e última seção apresenta as considerações finais.

3 Consiste numa atualização dos trabalhos de Peixoto (2008) e Peixoto et al. (2012), ampliando a amostra para todos os municípios brasileiros. 


\section{Material e métodos}

\subsection{Medidas de corrupção}

O Programa de Fiscalização de Municípios Pequenos e Médios por meio de Sorteios Públicos foi criado em 2003 com o objetivo de aumentar a transparência e melhorar a gestão pública brasileira.

A seleção dos municípios a serem fiscalizados ocorre por sorteio empreendido pela Caixa Econômica Federal, sendo assim totalmente aleatória. Para garantir isenção de ingerência política, ou de qualquer outra interferência no processo de decisão de escolha, os sorteios são acompanhados por representantes da sociedade civil e efetuados pelos mesmos equipamentos utilizados nas loterias.

Os dois primeiros sorteios foram realizados em caráter experimental, sendo nas seis loterias subsequentes adotadas uma série de mudanças. Foi apenas a partir do décimo sorteio que ficou instituído que 60 municípios seriam sorteados em cada loteria, sendo um número fixo de municípios sorteados por estado. ${ }^{4}$ Os municípios já sorteados podem ser novamente sorteados após um ano de carência.

Inicialmente os sorteios eram mensais, mas a frequência logo diminuiu (a média anual era de seis sorteios até 2005). Depois de 2005 o número de sorteios caiu pela metade. Desse modo, para cada ano pode ter ocorrido mais de um sorteio. Além disso, no começo eram elegíveis ao sorteio municípios com menos de 300 mil habitantes, mas a partir da nona loteria passaram a ser passíveis de fiscalização os municípios com menos de 500 mil habitantes (com exceção de capitais). ${ }^{5}$

Uma vez sorteado, o município recebe a visita dos auditores. Se o município tem até 20 mil habitantes, todas as funções de despesa são avaliadas. Se o município tem população entre 20 e 100 mil habitantes, além das áreas de educação, saúde e assistência social, outro grupo de despesa é sorteado e fiscalizado. Se o município tem mais de 100 mil habitantes fiscaliza-se uma função entre educação, saúde e assistência social.

4 O número de municípios que são sorteados a cada sorteio também sofreu alterações durante os primeiros anos, porém, após 2004, não houve mais alterações.

5 De acordo com o Censo 2010, somente 38 dos 5.565 municípios brasileiros tinham população superior a 500.000 habitantes, de tal forma que os municípios elegíveis ao programa representam quase a totalidade dos municípios. 
Os auditores fiscalizam o uso dos recursos com visitas às obras (para ver se o projeto está de acordo com o planejado) e análise de documentos fornecidos pela própria prefeitura (notas fiscais, processos de licitação, balanços, controles de entrada e saída de bens). Após a auditoria, são elaborados relatórios descrevendo as irregularidades encontradas, sendo posteriormente disponibilizados no sítio eletrônico da CGU.

É importante ressaltar que os auditores apenas registram as ocorrências que estejam em desacordo com as normas estabelecidas para o recebimento das transferências do governo federal, ou seja, os relatórios descrevem as irregularidades encontradas na execução de programas federais implementados de forma descentralizada e com repasses vinculados à União, tais como os programas associados à estratégia de atenção básica de saúde. ${ }^{6}$

Tabela 1 Número de municípios sorteados, por unidade da federação

\begin{tabular}{|c|c|c|c|}
\hline Acre & 4 & Paraíba & 32 \\
\hline Alagoas & 21 & Paraná & 31 \\
\hline Amapá & 4 & Pernambuco & 32 \\
\hline Amazonas & 11 & Piaú & 31 \\
\hline Bahia & 52 & Rio de Janeiro & 11 \\
\hline Ceará & 30 & Rio Grande do Norte & 32 \\
\hline Espírito Santo & 10 & Rio Grande do Sul & 43 \\
\hline Goiás & 23 & Rondônia & 10 \\
\hline Maranhão & 32 & Roraima & 4 \\
\hline Mato Grosso & 13 & Santa Catarina & 25 \\
\hline Mato Grosso do Sul & 12 & São Paulo & 58 \\
\hline Minas Gerais & 74 & Sergipe & 12 \\
\hline Pará & 30 & Tocantins & 12 \\
\hline Total & & & 649 \\
\hline
\end{tabular}

Fonte: CGU.

A amostra é composta de 649 municípios de todas as unidades da federação, sorteados e fiscalizados entre maio de 2007 e julho de 2010.?

6 Estes relatórios também foram utilizados por Mendes (2004) para avaliar as irregularidades na administração do Fundo de Manutenção e Desenvolvimento do Ensino Fundamental e de Valorização do Magistério (FUNDEF); por Ferraz e Finnan (2008) para avaliar a relação entre corrupção e eleições nos municípios; e por Brollo (2009) para mostrar o impacto da corrupção a nível municipal sobre as transferências recebidas do governo central.

7 Em alguns destes, nenhum dos três programas foram auditados, de modo que foram eli- 
A Tabela 1 descreve a distribuição dos municípios sorteados por estado.

A partir da leitura dos relatórios definimos as ocorrências, dentre todas as irregularidades reportadas, que entendemos corresponderem a atos que podem ser classificados como corruptos. A soma das irregularidades classificadas dessa maneira, definidas como o número total de ocorrências de corrupção, e denominadas a partir deste ponto apenas como irregularidades, consiste em nossa medida de corrupção.

A Tabela 2 mostra o número de ocorrências consideradas irregulares no Programa de Saúde da Família, para cada um dos anos da amostra. $O$ maior número das ocorrências refere-se às contratações irregulares de profissionais para a composição das Equipes de Saúde da Família (239 em 684 ou $35 \%$ ), seguida da falta de comprovação de despesas realizadas $(17,6 \%)$ e da irregularidade no gerenciamento de medicamentos e materiais $(17,3 \%)$.

Tabela 2 Irregularidades detectadas no Programa de Saúde da Família

\begin{tabular}{lrrrr|r|r}
\hline Ocorrência & $\mathbf{2 0 0 7}$ & $\mathbf{2 0 0 8}$ & $\mathbf{2 0 0 9}$ & $\mathbf{2 0 1 0}$ & Total \\
\hline $\begin{array}{l}\text { Irregularidades na contratação de profissionais ou na } \\
\text { constituição das ESF }\end{array}$ & 93 & 14 & 85 & 47 & 239 \\
\hline $\begin{array}{l}\text { Impossibilidade ou ausência de comprovação das } \\
\text { despesas realizadas a partir de recursos da conta PSF }\end{array}$ & 35 & 6 & 54 & 26 & 121 \\
\hline $\begin{array}{l}\text { Irregularidades no gerenciamento de medicamentos e } \\
\text { de materiais para uso exclusivo das ESF }\end{array}$ & 16 & 10 & 54 & 39 & 119 \\
\hline $\begin{array}{l}\text { Desvio de finalidade na aplicação de recursos finan- } \\
\text { ceiros destinados ao PACS/PSF }\end{array}$ & 53 & 7 & 24 & 15 & 99 \\
\hline $\begin{array}{l}\text { Inconsistência nos dados do SIAB } \\
\text { Irregularidades em licitações }\end{array}$ & 32 & 7 & 20 & 8 & 67 \\
\hline $\begin{array}{l}\text { Contratação irregular de organização executora do } \\
\text { programa }\end{array}$ & 2 & 2 & 3 & 2 & 9 \\
\hline Comprovação das despesas com Notas Fiscais falsas & 1 & 1 & 0 & 0 & 2 \\
\hline
\end{tabular}

Fonte: Elaboração própria.

A Tabela 3 reporta as irregularidades detectadas no Programa de Atendimento Assistencial Básico (PAB). As três ocorrências mais frequentes referem-se a irregularidades dos instrumentos básicos do sistema de planejamento do SUS (20,3\%), ao desvio de finalidade na utilização de recursos 
destinados ao $\mathrm{PAB}(18,1 \%)$ e irregularidades acerca da composição e/ou funcionamento dos Conselhos Municipais de Saúde (17,5\%).

Tabela 3 Irregularidades detectadas no PAB - Atendimento Assistencial Básico nos Municípios Brasileiros

\begin{tabular}{|c|c|c|c|c|c|}
\hline Ocorrência & 2007 & 2008 & 2009 & 2010 & Total \\
\hline $\begin{array}{l}\text { Irregularidades referentes aos instrumentos básicos } \\
\text { do sistema de planejamento do SUS }\end{array}$ & 145 & 85 & 178 & 177 & 585 \\
\hline Desvio de finalidade na utilização de recursos do PAB & 136 & 82 & 128 & 177 & 523 \\
\hline $\begin{array}{l}\text { Irregularidades relativas à formação e/ou ao funcio- } \\
\text { namento do Conselho Municipal de Saúde (CMS) }\end{array}$ & 113 & 86 & 149 & 158 & 506 \\
\hline $\begin{array}{l}\text { Documentação com elementos insuficientes para } \\
\text { atestar a finalidade de uso de recursos do PAB/desor- } \\
\text { ganização documental }\end{array}$ & 79 & 52 & 105 & 97 & 333 \\
\hline $\begin{array}{l}\text { Gerenciamento inadequado dos recursos financeiros } \\
\text { e das contas do } P A B\end{array}$ & 51 & 58 & 86 & 92 & 287 \\
\hline Impropriedades em processos licitatórios & 74 & 53 & 66 & 53 & 246 \\
\hline $\begin{array}{l}\text { Aquisição de bens ou contratação de serviços em } \\
\text { desacordo com a Lei de Licitações }\end{array}$ & 42 & 30 & 38 & 39 & 149 \\
\hline Indícios de fraude em processos licitatórios & 20 & 18 & 24 & 19 & 81 \\
\hline $\begin{array}{l}\text { Irregularidades na contratação de profissionais e/ou } \\
\text { de instituições privadas responsáveis pela execução } \\
\text { de serviços básicos de saúde }\end{array}$ & 12 & 12 & 13 & 20 & 57 \\
\hline $\begin{array}{l}\text { Pagamentos acima do estipulado em contrato ou no } \\
\text { processo licitatório; pagamentos intempestivos }\end{array}$ & 10 & 10 & 7 & 8 & 35 \\
\hline Restrição à competitividade em certame licitatório & 9 & 4 & 8 & 6 & 27 \\
\hline $\begin{array}{l}\text { Licitação na modalidade convite com número de } \\
\text { propostas válidas inferior a três }\end{array}$ & 11 & 6 & 1 & 5 & 23 \\
\hline Comprovação das despesas com Notas Fiscais falsas & 8 & 2 & 4 & 0 & 14 \\
\hline $\begin{array}{l}\text { Impropriedades detectadas no gerenciamento de } \\
\text { bens móveis e serviços realizados com recursos do } \\
\text { PAB-fixo }\end{array}$ & 4 & 0 & 2 & 7 & 13 \\
\hline $\begin{array}{l}\text { Cobrança de consultas básicas aos usuários do SUS } \\
\text { por parte de entidade conveniada }\end{array}$ & 3 & 2 & 2 & 0 & 7 \\
\hline
\end{tabular}

Fonte: Elaboração própria.

Com relação às auditorias no programa de Estruturação da Rede de Serviços de Atenção Básica de Saúde, as irregularidades em processos licitatórios perfazem $25 \%$ das ocorrências, seguidas pelas irregularidades no gerenciamento dos bens adquiridos por convênio com a União $(17,5 \%)$ 
e pela documentação precária referente a convênio ou licitação $(16,3 \%)$ (Tabela 4). ${ }^{8}$

Tabela 4 Irregularidades no âmbito da Estruturação da Rede de Serviços de Atenção Básica de Saúde

\begin{tabular}{|c|c|c|c|c|c|}
\hline Ocorrência & 2007 & 2008 & 2009 & 2010 & Total \\
\hline Impropriedades em processos licitatórios & 62 & 35 & 93 & - & 190 \\
\hline $\begin{array}{l}\text { Irregularidades no gerenciamento dos bens adquiri- } \\
\text { dos mediante convênio com a União }\end{array}$ & 50 & 19 & 61 & - & 130 \\
\hline Documentação precária referente a convênio ou licitação & 32 & 39 & 50 & - & 121 \\
\hline $\begin{array}{l}\text { Descumprimento de exigências perante órgão conce- } \\
\text { dente previstas em contrato }\end{array}$ & 36 & 21 & 22 & - & 79 \\
\hline $\begin{array}{l}\text { Preços Unitários de itens contratados com valores } \\
\text { acima aos de mercado/superfaturamento }\end{array}$ & 27 & 11 & 17 & - & 55 \\
\hline Indícios de fraude em processos licitatórios & 20 & 8 & 18 & - & 46 \\
\hline $\begin{array}{l}\text { Licitação na modalidade convite com número de } \\
\text { propostas válidas inferior a três }\end{array}$ & 15 & 9 & 12 & - & 36 \\
\hline Restrição à competitividade em certame licitatório & 13 & 7 & 10 & - & 30 \\
\hline Irregularidades na execução e pagamento de obras & 14 & 4 & 12 & - & 30 \\
\hline $\begin{array}{l}\text { Aquisição de bens ou contratação de serviços em } \\
\text { desacordo com a Lei de Licitações }\end{array}$ & 12 & 3 & 11 & - & 26 \\
\hline
\end{tabular}

Fonte: Elaboração própria.

Sem sombra de dúvida, a classificação das irregularidades em atos corruptos foi a parte mais difícil da pesquisa. Os relatórios não possuem um padrão, o que faz com que a qualidade de cada relatório dependa de cada auditor, visto que uns são mais detalhistas do que outros. Além disso, a classificação é em grande parte subjetiva. ${ }^{9}$

O número total de irregularidades por município, consideradas atos de corrupção, foi então utilizado como variável explicativa.

\subsection{Medidas de descentralização}

A disponibilidade de uma medida adequada de descentralização é uma das

8 Não existem informações sobre auditoria neste programa para o ano de 2010.

9 Relatórios disponíveis em http://www.cgu.gov.br. 
principais dificuldades encontradas na comparação de estruturas governamentais verticais (federações).

A literatura sobre descentralização fiscal, em geral aplicada a estudos comparando países, utiliza como fonte de dados o Government Finance Statistics (GFS) do Fundo Monetário Internacional, de modo que a medida geral de descentralização é dada pela parcela do gasto (ou da receita) subnacional no gasto (ou receita) governamental total ou no produto interno bruto.

Esta medida, contudo, apresenta dois problemas: i) as restrições fiscais impostas pelo governo central sobre os níveis de governo subnacional e a coordenação entre os níveis de governo na provisão de bens públicos e no financiamento conjunto não podem ser mensuradas quantitativamente; ii) elimina detalhes sobre o desenho dos sistemas fiscais, não permitindo identificar o grau de autonomia do gasto local, nem distinguir as fontes de receitas entre tributárias versus não tributárias, transferências intergovernamentais versus outras transferências, além de não separar quanto das transferências é condicional e que parcela é destinada a propósitos gerais (não condicionais).

Kaufman (1963) apresenta vários indicadores que medem o grau de controle do governo central sobre o governo subnacional, dentre os quais o envolvimento na seleção de staff administrativo, a frequência da consulta e a extensão da supervisão. Smith (1997) e Bahl (1999) discutem exaustivamente os fatores que descrevem conjuntamente a extensão da descentralização fiscal: conselhos localmente eleitos, provisões institucionais, o tamanho e o número de autoridades subnacionais, estruturas organizacionais, indicadores fiscais de gasto, taxação e capacidades de endividamento. Como medidas alternativas de descentralização de natureza semelhante aparecem ainda o tamanho e o número de autoridades locais, o número de jurisdições locais normalizado pela população ou número de tiers do governo subnacional.

Dados esses problemas, novas abordagens que buscam levar em conta a estrutura vertical de tomada de decisão, apareceram para analisar o grau de descentralização fiscal.

Do lado da oferta de bens públicos, a extensão pela qual os governos subnacionais dependem de transferências do governo central para apoiar os seus gastos (desequilíbrio fiscal vertical) é usada como uma medida de autonomia de gasto. Uma abordagem mais exaustiva, como a de Pola (1999), envolve a separação dos gastos dos governos subnacionais por fun- 
ção e sua classificação de acordo com o grau de discricionariedade local na sua formulação e execução.

Do lado das receitas, o grau de dependência em relação aos recursos próprios (own revenue) é geralmente usado como um indicador de autonomia de receita dos governos subnacionais. Esse indicador é definido como a razão entre receitas totais líquidas de transferências intergovernamentais e a receita total dos governos locais.

Uma estrutura analítica mais recente, fornecida pela OCDE - Organização para Cooperação e Desenvolvimento Econômico (OECD, 1999, 2001) - que classifica os impostos de acordo com o grau de discricionariedade local, deu origem a outros indicadores de descentralização fiscal. A OCDE identifica três fontes de receitas subnacionais: receitas tributárias, não tributárias e transferências intergovernamentais. As receitas tributárias e as transferências intergovernamentais são subdivididas em dois grupos. Primeiro, caso os governos subnacionais tenham controle significativo ou total sobre um imposto (um controle próprio sobre a alíquota ou sobre a base tributária de um imposto e sua alíquota) ele é classificado como "own tax revenue". Segundo, caso o governo subnacional não tenha controle ou tenha apenas controle limitado sobre a alíquota e a base de um imposto e o governo central determine a divisão das receitas, ele é classificado como "revenue from tax sharing". Receitas não-tributárias incluem rendimento de operações e propriedade de negócios, taxas, multas e tarifas administrativas.

As transferências intergovernamentais são posteriormente classificadas como de propósitos gerais ou específicos. As transferências com propósitos gerais podem ser usadas como receitas próprias, mas elas podem ser alocadas com base em critérios objetivos ou discricionários pelo governo central. As transferências específicas são carimbadas para determinados propósitos e a alocação pode ou não ser condicional por meio dos governos subnacionais. Portanto, transferências com propósitos gerais ou específicos são identificadas como subgrupos separados.

Baseado nesta estrutura da OCDE, Stegarescu (2004) constrói diferentes medidas de autonomia fiscal e descentralização de receitas. Por exemplo, uma de suas medidas do grau de autonomia fiscal é definida como a receita tributária própria do governo subnacional dividida pela receita tributária total do governo subnacional. Uma de suas medidas de descentralização da receita tributária é definida como receita tributária própria do governo subnacional dividida pela receita tributária do governo geral consolidado. 
Ebel e Yilmaz (2002) incluem transferências com propósitos gerais com critérios objetivos e transferências específicas não condicionais na variável de descentralização, uma vez que os governos subnacionais têm pelo menos alguma autonomia de gasto dessas transferências.

Para o Brasil, Guedes e Gasparini (2007), ao discutirem a relação entre descentralização e tamanho do governo, utilizam duas medidas de descentralização: a participação da receita tributária municipal nas despesas orçamentárias totais para captar a capacidade de autofinanciamento dos municípios e, seguindo a tradição na literatura, usam também a participação das despesas orçamentárias municipais nas despesas orçamentárias totais do governo.

Decidimos usar duas medidas de descentralização.

A primeira corresponde a uma medida "clássica" de descentralização, dada pela parcela dos gastos com saúde financiados com recursos próprios do município em relação ao gasto total com saúde na localidade. Para o caso particular deste trabalho, que trata exclusivamente do setor saúde e de unidades dentro do mesmo país, boa parte dos problemas desta medida fica minimizada, pois não se trata de gastos/receitas agregados dos países nos vários setores nem de localidades com diferentes estruturas e desenhos institucionais.

A segunda medida corresponde a uma proxy para descentralização administrativa, caracterizada pela adesão do município ao Pacto pela Saúde. O Pacto pela Saúde constitui a reunião de um conjunto de reformas institucionais, pactuada entre as três esferas de gestão do SUS, que procura estabelecer novos procedimentos de gestão. A adesão das entidades federativas (União, estados e municípios) ao Pacto pela Saúde envolve a assinatura do Termo de Compromisso de Gestão, que estabelece metas anuais e compromissos de gestão para cada agente responsável.

O Pacto possui três componentes: i) Pacto pela Vida, cujas prioridades estão voltadas para o fortalecimento da Atenção Básica à Saúde e prevenção; ii) Pacto em Defesa do SUS, que estabelece divisão de responsabilidades entre os entes federativos e a gestão compartilhada do SUS; iii) Pacto de Gestão, com diretrizes voltadas para a definição de responsabilidades, a descentralização e a regionalização do financiamento da saúde, além de estabelecer normas de participação social, planejamento e gestão do trabalho. A responsabilidade pela avaliação e fiscalização do Pacto pela Saúde é da Comissão Intergestores Tripartite (CIT), instância colegiada de "articu- 
lação, negociação e pactuação entre os gestores das três esferas de governo, para a regulamentação e operacionalização das políticas de saúde no âmbito do Sistema Único de Saúde" (BRASIL, 2009).

A Comissão Intergestores Tripartite (CIT) tem composição paritária formada por 18 membros, sendo seis indicados pelo Ministério da Saúde (MS), seis pelo Conselho Nacional de Secretários Estaduais de Saúde (Conass) e seis pelo Conselho Nacional de Secretários Municipais de Saúde (Conasems). A representação de estados e municípios nessa Comissão é regional, sendo um representante para cada uma das cinco regiões do país, além dos Presidentes de Conass e Conasems. As decisões são tomadas por consenso e não por votação. ${ }^{10}$

\section{Teoria e cálculos}

Dado que a variável dependente é o número de irregularidades classificadas como corrupção, utilizamos os modelos de contagem para estimar o impacto da descentralização sobre a corrupção, uma vez que a variável de interesse é um número inteiro não-negativo.

Embora a hipótese de Poisson seja bastante restritiva, assumimos inicialmente que o evento de interesse apresenta um processo gerador que obedece a uma distribuição de Poisson. Diante disso, a probabilidade de se observarem $y$ ocorrências do evento $Y$ é dada por:

$$
\operatorname{Pr}[Y=y]=\frac{e^{-\mu} \mu^{y}}{y !}, y \in \mathbb{Z}^{+}
$$

onde $\mu$ é a taxa de intensidade do evento, sendo $E(Y)=\mu$ e $\operatorname{Var}(Y)=\mu$. A igualdade entre a variância e a média é denominada equidispersão.

O modelo de regressão de Poisson deriva da parametrização da taxa de intensidade do evento, $\mu$, com relação a um vetor de variáveis independentes, $X$, com a constante inclusa. Adotando-se a hipótese usual de uma especificação exponencial, temos, para cada uma das $i=1, \ldots, N$ observações:

$$
\mu_{i}=\exp \left(X_{i}^{\prime} \beta\right)
$$

10 Fonte: Ministério da Saúde (www.saude.gov.br). 
É importante observar que, dada a propriedade de equidispersão, a regressão de Poisson apresenta heterocedasticidade por construção, já que $\operatorname{Var}\left(y_{i} \mid X_{i}\right)=\exp \left(X_{i}^{\prime} \beta\right)$.

Sob a hipótese de $y_{i} \mid X_{i}^{\prime} \sim$ iid, tem-se a função de verossimilhança:

$$
L(\beta)=\prod_{i=1}^{N} \frac{e^{-\mu_{i}} \mu_{i}^{y_{i}}}{y_{i} !}
$$

Substituindo (2) em (1), obtém-se a função de log-verossimilhança dada por:

$$
\ln L(\beta)=\sum_{i=1}^{N}\left[y_{i} X_{i}^{\prime} \beta-\exp \left(X_{i}^{\prime} \beta\right)-\ln \left(y_{i} !\right)\right]
$$

O estimador de Poisson de máxima verossimilhança (MLE) é a solução de um sistema de equações composto pelas condições de primeira ordem, ${ }^{11}$ que pode ser resolvido por métodos numéricos:

$$
\sum_{\mathrm{i}=1}^{\mathrm{N}}\left[y_{\mathrm{i}}-\exp \left(X_{\mathrm{i}}^{\prime} \beta\right)\right] X_{\mathrm{i}}^{\prime}=0
$$

São duas as principais restrições do modelo de Poisson. Em primeiro lugar, como a distribuição é condicionada a um único parâmetro, a taxa de incidência $\mu$, todos os momentos da variável dependente são função deste mesmo parâmetro. Assim, os parâmetros de posição e de dispersão são sintetizados apenas em $\mu .{ }^{12}$ Em segundo lugar, a distribuição de Poisson não lida com a sobredispersão (overdispersion) dos dados, ou seja, a variância usualmente é maior que a média que usualmente caracteriza os dados de contagem. Sob a hipótese de que o modelo está especificado corretamente, a hipótese de equidispersão não afeta a consistência do estimador de máxima verossimilhança, mas ele deixa de ser eficiente.

Sendo assim, depois de estimar um modelo de Poisson é importante testar a hipótese de igualdade entre a média e a variância de $y$. Cameron e Trivedi (1990) propuseram um teste baseado na seguinte especificação:

11 Cameron e Trivedi (2005, p. 668). Estes autores também trazem as formas funcionais para a variância do estimador.

12 Esta limitação se torna relevante diante do problema do excesso de zeros (Cameron; Trivedi, 2005), isto é, a distribuição de Poisson prevê, em muitos casos, uma probabilidade de se observar $y=0$ menor que a observada de fato na amostra. 


$$
\operatorname{Var}\left[y_{i} \mid X_{i}\right]=\mu_{i}+\delta g\left(\mu_{i}\right)
$$

na qual se testa $H_{0}: \delta=0$ contra a hipótese alternativa $H_{1}: \delta>0$ (sobredispersão) ou $H_{1}: \delta<0$ (subdispersão).

Em síntese, a partir de um modelo de Poisson são construídos os valores estimados $\hat{\mu}_{i}=\exp \left(X_{i}^{\prime} \beta\right)$. A estatística do teste, $z_{i}$, é definida como:

$$
z_{i}=\frac{\left(y_{i}-\hat{\mu}_{i}\right)^{2}-y_{i}}{\hat{\mu}_{i} \sqrt{2}}
$$

A ideia do teste é verificar a significância de $z_{i}$ em relação ao valor esperado de uma regressão baseada no modelo de Poisson. A estatística é assintoticamente normal sob a hipótese nula (Cameron; Trivedi, 1990).

Diante da ausência de equidispersão, a alternativa é usar outras distribuições para dados de contagem. Assim, apresentamos também os resultados para a distribuição binomial negativa, na qual a dispersão é modelada como função da média esperada. Os dois primeiros momentos da Binomial Negativa são dados por (Cameron; Trivedi, 2005, p. 676):

$$
\begin{aligned}
& E[y \mid \mu, \alpha]=\mu \\
& \operatorname{Var}[y \mid \mu, \alpha]=\mu(1+\alpha \mu)
\end{aligned}
$$

onde $\alpha$ é um parâmetro a ser estimado conjuntamente com as estimativas por máxima verossimilhança.

\section{Resultados}

O Gráfico 1 apresenta o histograma do número de irregularidades presente no banco de dados, sendo a média igual a 6,64, para uma mediana de 6, moda igual a 8 e um máximo de 31 . Há 45 municípios para os quais não foram identificadas irregularidades. 
Gráfico 1 Histograma das irregularidades identificadas

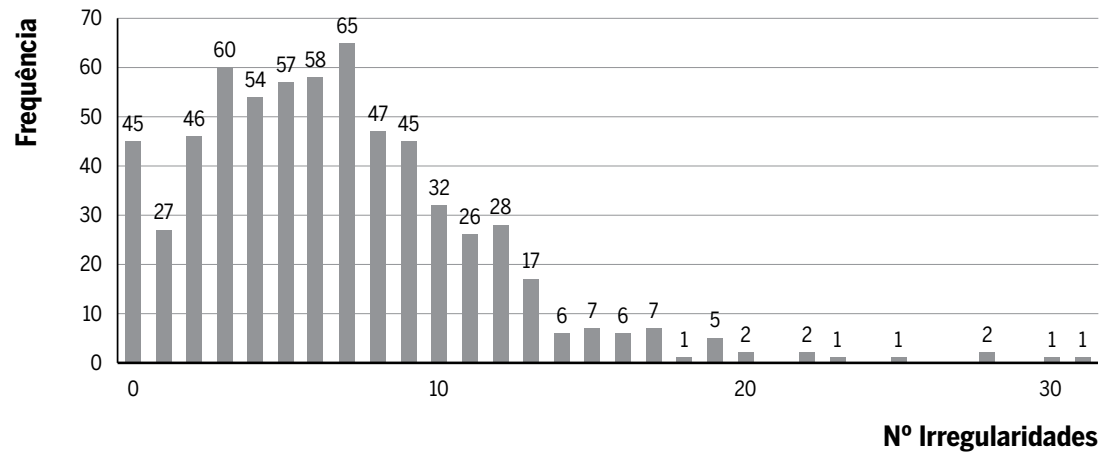

Fonte: Elaboração própria.

A Tabela 5 reporta das irregularidades segundo a Unidade da Federação. Os estados cujos municípios apresentam maior média de ocorrências são Rondônia e Roraima (média de 9), ao passo que o Piauí é o que registra a menor média $(4,13)$.

Tabela 5 Número de irregularidades identificadas nos relatórios da CGU, por Unidade da Federação

\begin{tabular}{lr|r|r|r|r}
\hline UF & $\begin{array}{r}\mathbf{N}^{\mathbf{0}} \text { municípios } \\
\text { na amostra }\end{array}$ & $\begin{array}{r}\text { Média de } \\
\text { irregularidades }\end{array}$ & $\begin{array}{r}\text { Desvio } \\
\text { padrão }\end{array}$ & Mínimo & Máximo \\
\hline AC & 4 & 5.75 & 6.13 & 0 & 12 \\
\hline AL & 21 & 7.71 & 5.23 & 0 & 25 \\
\hline AM & 11 & 5.64 & 2.80 & 1 & 9 \\
\hline AP & 4 & 7.00 & 2.16 & 4 & 9 \\
\hline BA & 52 & 7.38 & 5.01 & 0 & 20 \\
\hline CE & 30 & 8.07 & 6.68 & 0 & 31 \\
\hline ES & 10 & 8.00 & 6.34 & 0 & 19 \\
\hline GO & 23 & 6.04 & 3.78 & 0 & 14 \\
\hline MA & 32 & 8.59 & 6.51 & 0 & 28 \\
\hline MG & 74 & 5.77 & 3.73 & 0 & 13 \\
\hline MS & 12 & 7.42 & 8.21 & 1 & 30 \\
\hline MT & 13 & 5.38 & 2.53 & 1 & 9 \\
\hline PA & 30 & 6.63 & 4.63 & 0 & 19 \\
\hline PB & 32 & 7.19 & 4.29 & 0 & 17 \\
\hline PE & 32 & 7.56 & 4.70 & 0 & 23 \\
\hline
\end{tabular}




\begin{tabular}{l|r|r|r|r|r}
\hline UF & $\begin{array}{r}\mathbf{N}^{\mathbf{0}} \text { municípios } \\
\text { na amostra }\end{array}$ & $\begin{array}{r}\text { Média de } \\
\text { irregularidades }\end{array}$ & $\begin{array}{r}\text { Desvio } \\
\text { padrão }\end{array}$ & Mínimo & Máximo \\
\hline PI & 31 & 4.13 & 2.87 & 0 & 10 \\
\hline PR & 31 & 5.84 & 3.47 & 0 & 17 \\
\hline RJ & 11 & 6.00 & 5.02 & 0 & 14 \\
\hline RN & 32 & 7.09 & 4.31 & 0 & 16 \\
\hline RO & 10 & 9.00 & 6.24 & 1 & 22 \\
\hline RR & 4 & 9.00 & 3.16 & 6 & 13 \\
\hline RS & 43 & 6.02 & 3.72 & 0 & 15 \\
\hline SC & 25 & 6.20 & 4.84 & 0 & 19 \\
\hline SE & 12 & 5.50 & 5.00 & 1 & 20 \\
\hline SP & 58 & 6.28 & 4.56 & 0 & 19 \\
\hline
\end{tabular}

Fonte: Elaboração própria.

O PIB municipal per capita de 2007, a população de 2007 e o grau de urbanização (definido pela percentagem de população urbana no Censo de 2000) foram usados como variáveis de controle (fonte: IBGE). Tais variáveis visam controlar as características socio-econômicas do município, como sua capacidade fiscal e seu tamanho. Também foram incluídas dummies de ano, de partido político e de unidade da federação, com o objetivo de controlar, respectivamente, os choques macroeconômicos nacionais e o perfil político do município, o qual pode afetar a ocorrência de irregularidades. As dummies de Unidade da Federação visam controlar por efeitos fixos estaduais.

A título de controle para a situação fiscal do município, adicionaram-se as transferências do SUS. Os municípios são obrigados por lei a destinar ao menos $15 \%$ de suas receitas para o financiamento da saúde. Além disso, o governo federal complementa os recursos municipais com transferências fundo a fundo, ou seja, por meio de transferências automáticas do Fundo Nacional de Saúde para os fundos municipais de saúde. A autonomia de gastos (e, portanto, de desvios de recursos) é baixa, tendo em vista que essas transferências devem ser, obrigatoriamente, destinadas ao financiamento de programas preestabelecidos pelo governo federal.

A Tabela 6 apresenta as estatísticas básicas das irregularidades segundo o partido político do prefeito do município sorteado. As prefeituras administradas pelo PV apresentam maior média de registro de irregulari- 
dades (9.0), seguidas do PSC (8.89). Mesmo assim, não se nota nenhum padrão específico por partido político.

Tabela 6 Número de irregularidades identificadas nos relatórios da CGU, por partido do prefeito

\begin{tabular}{lrrrrr|r}
\hline Partido & $\begin{array}{r}\mathbf{N}^{0} \text { municípios } \\
\text { na amostra }\end{array}$ & $\begin{array}{r}\text { Média de } \\
\text { irregularidades }\end{array}$ & $\begin{array}{r}\text { Desvio } \\
\text { padrão }\end{array}$ & Mínimo & Máximo \\
\hline DEM & 30 & 7.43 & 6.18 & 0 & 30 \\
\hline PC do B & 7 & 7.71 & 5.91 & 0 & 17 \\
\hline PDT & 39 & 7.54 & 3.73 & 0 & 17 \\
\hline PFL & 41 & 5.73 & 3.11 & 0 & 12 \\
\hline PHS & 4 & 7.75 & 4.65 & 2 & 12 \\
\hline PL & 20 & 6.15 & 3.70 & 1 & 17 \\
\hline PMDB & 119 & 6.96 & 5.03 & 0 & 31 \\
\hline PMN & 3 & 4.00 & 3.00 & 1 & 7 \\
\hline PP & 61 & 6.02 & 4.49 & 0 & 20 \\
\hline PPS & 28 & 4.86 & 3.88 & 0 & 14 \\
\hline PR & 35 & 6.49 & 3.41 & 0 & 12 \\
\hline PRB & 4 & 7.00 & 4.69 & 4 & 14 \\
\hline PRP & 4 & 5.00 & 2.83 & 1 & 7 \\
\hline PRTB & 2 & 6.00 & 1.41 & 5 & 7 \\
\hline PSB & 32 & 5.69 & 4.37 & 0 & 16 \\
\hline PSC & 9 & 8.89 & 7.83 & 3 & 28 \\
\hline PSDB & 81 & 6.98 & 4.87 & 0 & 23 \\
\hline PSL & 4 & 4.00 & 4.69 & 1 & 11 \\
\hline PT & 54 & 7.24 & 5.64 & 0 & 28 \\
\hline PT do B & 4 & 4.75 & 2.22 & 2 & 7 \\
\hline PTB & 49 & 6.69 & 4.93 & 0 & 25 \\
\hline PTC & 2 & 4.00 & 4.24 & 1 & 7 \\
\hline PV & 11 & 9.00 & 4.22 & 4 & 17 \\
\hline FV & & & & &
\end{tabular}

Fonte: Elaboração própria.

A Tabela 7 sumariza as médias e os desvios-padrão das variáveis independentes. Em média, 55,3\% do financiamento municipal da saúde é realizado com recursos próprios. Além disso, como se pode observar, cerca de $43 \%$ dos municípios sorteados aderiram ao Pacto pela Saúde, gerido pela Comissão Intergestores Tripartite. 
Tabela 7 Estatísticas descritivas - variáveis independentes

\begin{tabular}{lrrr}
\hline Variável & Média & Desvio padrão \\
\hline Descentralização Financeira (\%) & 55,30 & 23,47 \\
\hline Descentralização Administrativa (dummy) & 0,43 & 0,50 \\
\hline Receita Transferência SUS (R\$ mil) & $2.877,71$ & $9.125,09$ \\
\hline PIB 2007 (R\$) & $4.369,86$ & $4.027,98$ \\
\hline População 2007 & $26.573,29$ & $58.774,38$ \\
\hline \% População Urbana & 56,72 & 23,00 \\
\hline
\end{tabular}

Fonte: Elaboração própria.

Os resultados das estimações do modelo de Poisson são apresentados na Tabela 8. A especificação (1) considera a medida de Descentralização Administrativa, enquanto a especificação (2) utiliza a medida de Descentralização Financeira.

Encontra-se evidência de que a descentralização administrativa atua no sentido de reduzir a corrupção, tendo em vista que o coeficiente dessa variável é negativo e estatisticamente significante ao nível de $5 \% .{ }^{13}$ Conforme esperado, quanto maior o PIB per capita do município, menor o número de irregularidades (significativo a 5\%) e o tamanho populacional do município não tem impacto significativo no montante de ocorrências. A Descentralização Financeira, por outro lado, parece não exercer influência significativa sobre a corrupção.

Tabela 8 Irregularidades e descentralização - Poisson

\begin{tabular}{l|r|r}
\hline \multirow{2}{*}{ Variáveis } & \multicolumn{2}{r}{ Especificação } \\
\cline { 2 - 3 } & $\mathbf{( 1 )}$ & $\mathbf{( 2 )}$ \\
\hline \multirow{2}{*}{ Descentralização Administrativa } & ${ }^{* *}-0.145$ & - \\
\cline { 2 - 3 } & $(0.0719)$ & -0.149 \\
\hline \multirow{2}{*}{ Descentralização Financeira } & - & $(0.137)$ \\
\cline { 2 - 3 }
\end{tabular}

13 Neste artigo, não tratamos potenciais problemas de endogeneidade que podem decorrer da existência de correlação entre a decisão de aderir ao Pacto da Saúde e a ocorrência de corrupção em si. Adotamos esta posição em função da ambiguidade de potenciais vieses. Por exemplo, municípios com maior propensão à corrupção podem ser menos propensos a aderir, o que acarretaria um viés de superestimação do real impacto. A não adesão estaria associada ao menor engajamento e preocupação dos gestores em relação ao provimento dos serviços de saúde que por sua vez pode estar associada a uma maior propensão à corrupção. Por outro lado, a adesão ao Pacto está condicionada ao acesso de mais recursos que poderiam ser passíveis de corrupção, tornando as prefeituras mais corruptas mais propensas à adesão, ocasionando um viés de subestimação do impacto. 


\begin{tabular}{|c|c|c|}
\hline \multirow{2}{*}{ Variáveis } & \multicolumn{2}{|c|}{ Especificação } \\
\hline & (1) & (2) \\
\hline \multirow{2}{*}{ Log PIB per capita 2007} & ** -0.110 & **-0.0993 \\
\hline & $(0.0463)$ & $(0.0494)$ \\
\hline \multirow{2}{*}{ Log População 2007} & 0.0413 & 0.0325 \\
\hline & $(0.0326)$ & $(0.0329)$ \\
\hline \multirow{2}{*}{ Receitas Transferências SUS } & $2.76 \mathrm{e}-09$ & $2.57 e-09$ \\
\hline & $(3.84 \mathrm{e}-09)$ & $(3.94 \mathrm{e}-09)$ \\
\hline \multirow{2}{*}{ População Urbana (\%) } & 0.0500 & 0.0213 \\
\hline & $(0.132)$ & $(0.130)$ \\
\hline \multirow{2}{*}{ Dummy ano 2007} & ${ }^{* *} 0.195$ & $* * * 0.315$ \\
\hline & $(0.0913)$ & $(0.0859)$ \\
\hline \multirow{2}{*}{ Dummy ano 2009} & 0.122 & ${ }^{* *} 0.189$ \\
\hline & $(0.0871)$ & $(0.0904)$ \\
\hline \multirow{2}{*}{ Dummy ano 2010} & $* * * 0.254$ & $* * * 0.308$ \\
\hline & $(0.0698)$ & $(0.0748)$ \\
\hline Dummies de Unidade da Federação (§) & Sim & Sim \\
\hline Dummies de Partido Político do Prefeito (§) & $\operatorname{Sim}$ & Sim \\
\hline Constante & $* * * 1.820$ & $* * * 1.888$ \\
\hline Observações & 640 & 640 \\
\hline Teste de equidispersão (estatística t) & $* * * 9.76$ & $* * * 9.41$ \\
\hline
\end{tabular}

Desvios-padrão robustos entre parênteses. $\left({ }^{* *}\right)$ Significativo a 1\%; $\left({ }^{* *}\right)$ Significativo a 5\%; $\left({ }^{*}\right)$ Significativo a 10\%. (§) As dummies de Unidade da Federação e de partidos políticos mostraram significância conjunta pelo teste de razão de verossimilhança de restrição de parâmetros. Para ambas as especificações, a estatística $\chi^{2}$ com 52 graus de liberdade mostrou-se significativa a $1 \%$.

A última linha da Tabela 8 traz a estatística t do teste para equidispersão, a qual revela a rejeição da hipótese nula de que a variância e a média são iguais, o que faz com que o modelo de Poisson não seja a especificação correta.

Passamos, então, à estimação de um modelo binomial negativo cujos resultados são reportados na Tabela 9. Como se pode observar, os resultados referentes ao impacto da descentralização sobre o número de ocorrências indicativas de corrupção são análogos ao caso da distribuição de Poisson, isto é, a adesão ao Pacto pela Saúde está negativamente associada à ocorrência de irregularidades, ao passo que a proporção de recursos municipais no financiamento da saúde não se revelou significativo. Os sinais das variáveis 
de controle também não se alteraram.

Tabela 9 Irregularidades e descentralização - Binomial Negativa

\begin{tabular}{|c|c|c|}
\hline \multirow{2}{*}{ Variáveis } & \multicolumn{2}{|c|}{ Especificação } \\
\hline & (1) & (2) \\
\hline \multirow{2}{*}{ Descentralização administrativa } & ${ }^{* *}-0.143$ & \\
\hline & $(0.0664)$ & \\
\hline \multirow{2}{*}{ Descentralização financeira } & & -0.0968 \\
\hline & & $(0.142)$ \\
\hline \multirow{2}{*}{ Log PIB per capita 2007} & $* *-0.116$ & $* *-0.111$ \\
\hline & $(0.0458)$ & $(0.0500)$ \\
\hline \multirow{2}{*}{ Log População 2007} & 0.0486 & 0.0423 \\
\hline & $(0.0347)$ & $(0.0354)$ \\
\hline \multirow{2}{*}{ Receitas Transferências SUS } & $1.33 \mathrm{e}-09$ & $1.66 \mathrm{e}-09$ \\
\hline & $(4.87 e-09)$ & $(5.05 e-09)$ \\
\hline \multirow{2}{*}{ População Urbana (\%) } & 0.0360 & 0.0108 \\
\hline & $(0.136)$ & $(0.135)$ \\
\hline \multirow{2}{*}{ Dummy ano 2007} & ${ }^{* *} 0.187$ & $* * * 0.295$ \\
\hline & $(0.0869)$ & $(0.0844)$ \\
\hline \multirow{2}{*}{ Dummy ano 2009} & 0.134 & ${ }^{* *} 0.185$ \\
\hline & $(0.0865)$ & $(0.0913)$ \\
\hline \multirow{2}{*}{ Dummy ano 2010} & $* * 0.264$ & $* * * 0.300$ \\
\hline & $(0.0690)$ & $(0.0734)$ \\
\hline Dummies de UF & $\operatorname{Sim}$ & Sim \\
\hline Dummies de Partido do Prefeito & Sim & Sim \\
\hline \multirow{2}{*}{ Constante } & $* * * 1.771$ & $* * * 1.805$ \\
\hline & $(0.427)$ & $(0.452)$ \\
\hline \multirow{2}{*}{ Ln(Alpha) $)^{14}$} & $* * *-1.203$ & $* * *-1.192$ \\
\hline & $(0.104)$ & $(0.104)$ \\
\hline Alpha & 0.300 & 0.304 \\
\hline Observações & 639 & 639 \\
\hline
\end{tabular}

Desvios-padrão robustos entre parênteses. $\left({ }^{* *}\right)$ Significativo a 1\%; $\left({ }^{* *}\right)$ Significativo a 5\%; $\left({ }^{*}\right)$ Significativo a 10\%. As dummies de Unidade da Federação e de partidos políticos mostraram significância conjunta pelo teste de razão de verossimilhança de restrição de parâmetros. Para ambas as especificações, a estatística $\chi^{2}$ com 52 graus de liberdade mostrou-se significativa a $1 \%$.

$14 \bigcirc$ modelo de regressão segundo a distribuição negativa binomial parametriza a variância como função de $1+\alpha \exp \left\{X_{i}^{\prime} \hat{\beta}\right\}$. Ver detalhes em Cameron e Trivedi (2005). 


\section{Considerações finais}

O objetivo do artigo foi avaliar o impacto da descentralização sobre a corrupção na área de saúde no território brasileiro. Como medida de corrupção foi usado o número de irregularidades passíveis de serem associadas com atos corruptos, contabilizadas a partir dos relatórios da CGU no âmbito do Programa de Fiscalização de Municípios Pequenos e Médios por meio de Sorteios Públicos.

Os resultados sugerem que a descentralização administrativa reduz o número de ocorrências de irregularidades, enquanto descentralização financeira não afeta significativamente o nível de corrupção.

Peixoto et al. (2012) também procuram verificar o efeito de dois indicadores de descentralização sobre a ocorrência de irregularidades nas auditorias da CGU: uma medida de descentralização financeira, construída pela proporção do gasto em saúde sob responsabilidade municipal; e uma medida de descentralização administrativa, definida pelo percentual de produção de serviços ambulatoriais sob gestão municipal. A amostra utilizada compreende, no entanto, somente cerca de 160 municípios do Sudeste do Brasil sorteados entre 2004 e 2006. Os resultados encontrados não indicam relação entre corrupção e descentralização, qualquer que seja a medida de descentralização utilizada.

Assim, ao ampliar a amostra para todos os municípios brasileiros entre 2007 e 2010, os resultados com relação à descentralização financeira se mantêm. Entretanto, o efeito da descentralização administrativa se mostra estatisticamente significante e favorável, em linha com o obtido por Goel e Nelson (2011) para os Estados Unidos. Para o caso do Brasil, como a descentralização de saúde pode ser considerada específica, as evidências encontradas são coerentes com os resultados desses autores. A saber, a descentralização pode gerar benefícios conforme o tipo de descentralização. Assim, é possível obter níveis de corrupção reduzidos em administrações locais de saúde primária associada à consequente melhora na qualidade de oferta desses bens por meio da responsabilidade local em ofertar serviços de saúde.

Além disso, os resultados também refletem o fato da descentralização financeira ter evoluído muito pouco ao longo do período investigado quando comparado com a evolução da descentralização administrativa no mesmo período. A aceleração da descentralização administrativa foi maior nos últimos anos da primeira década do século XXI do que nos primeiros 
anos. Notadamente, este estudo investiga o período de 2007 a 2010 enquanto Peixoto et al. (2012) investigam o período de 2004 a 2006.

Este trabalho possui limitações decorrentes da base de dados e das técnicas utilizadas. Extensões futuras são possíveis, como a ampliação da base de dados e o tratamento de potenciais problemas de endogeneidade que podem surgir do fato da decisão de aderir ao Pacto da Saúde ser correlacionada com a corrupção. Mesmo assim, as evidências deste artigo contribuem para corroborar a relação negativa entre descentralização administrativa e corrupção. As evidências trazidas sugerem o desenvolvimento de políticas de incentivo à descentralização administrativa, via adesão ao Pacto pela Saúde. Trata-se, ao que tudo indica, de um importante fator para reduzir a ocorrência de irregularidades que podem, em alguns casos, caracterizar práticas ilegais ou corruptas.

\section{Referências}

BAHL, R. Implementation rules for fiscal decentralization, Andrew Young School of Policy Studies, Georgia State University, unpublished manuscript, 1999.

BRASIL. Portaria Ministério da Saúde no 2.670/2009. Aprova o Regimento Interno da Comissão Intergestores Tripartite - CIT. 2009.

BRENNAN, G.; BUCHANAN, J.. The power to tax. New York: Cambridge University Press, 1980.

BROLLO, F. Who is punishing corrupt politicians - Voters or the government? Evidence from the Brazilian anti-corruption program, 2009. Disponível em: http://www.bu.edu/econ/files/2012/11/dp168.pdf.

BRUECKNER, J. Fiscal decentralization in LDCs: the effects of local corruption and tax evasion. Department of Economics, University of Illinois at Urbana-Champaign, 1999.

CAMERON, A. C.; TRIVEDI, P.K. Regression-based tests for overdispersion in the Poisson model. Journal of Econometrics, Elsevier, v. 46, n.3, p. 347-364, December, 1990.

CAMERON, A. C.; TRIVEDI, P.K. Microeconometrics. New York: Cambridge University Press, 2005.

CAREAGA, M.; WEINGAST, B. The fiscal pact with the devil: a positive approach to fiscal federalism, revenue sharing, and good governance. Working paper. Hoover Institution, Stanford University, 2000.

EBEL, R.; YILMAZ, S. On the measurement and impact of fiscal decentralization. Policy Research Working Paper 2809. World Bank, 2002. Disponível em: http://documents.worldbank.org/curated/en/309771468764353136/pdf/multi0page.pdf. Acesso em: 20/04/2017.

FAN, C. S.; LIN, C.; TREISMAN, D. Political decentralization and corruption: Evidence from around the world. Journal of Public Economics, 93, p. 14-34, 2009. 
FERRAZ, C.; FINAN, F. Exposing corrupt policiticans: the effects of Brazil's publicily released audits on electoral outcomes. Quarterly Journal of Economics, v.123, n.2, p. 703-745, 2008.

FISMAN, R.; GATTI, R. Decentralization and corruption: evidence across countries. Journal of Public Economics, 83, p. 325-346, 2002a.

FISMAN, R.; GATTI, R. Decentralization and corruption: evidence from U.S. federal transfer programs. Public Choice, v.113, p. 25-35, 2002 b.

FREDRIKSSON, P. G.; VOLLEBERGH, H. R. J. Corruption, federalism, and policy formation in the OECD: the case of energy policy. Public Choice, v. 140, n. 1-2, p. 205-221, 2009.

GOEL, R. K; NELSON, M. A. Government fragmentation versus fiscal decentralization and corruption. Public Choice, v. 148, p. 471-490, 2011.

GUEDES, K.P.; GASPARINI, C.E. Descentralização fiscal e tamanho do governo no Brasil, Economia Aplicada, v. 11, n. 2, p. 303-323, 2007.

KAUFMAN, H. Politics and policies in state and local governments. Englewood Cliffs (N.J.), 1963.

MENDES, M. Análise das irregularidades na administração municipal do FUNDEF: constatações do programa de fiscalização a partir de sorteios públicos da Controladoria Geral da União. Transparência Brasil, Dezembro, 2004.

OECD. Fiscal design across levels of government, Paris, 2001. Disponível em: https://www.oecd. org/tax/tax-policy/1907023.pdf. Acesso em 20/04/2017.

OECD. Taxing powers of state and local government. OECD Tax Policy Studies, n. 7, Paris, 1999.

POLA, G. A comparative view of local finances in EU member countries: are there any lessons to be drawn? In: FOSSATI, A.; PANELLA, G. (Orgs.) Fiscal federalism in the European Union. London and New York: Routledge, p. 15-62, 1999.

PEIXOTO, S. G. D. Eficácia e gestão da Política de Atenção Básica de Saúde nos municípios brasileiros. 2008. Dissertação de Mestrado, IPE/Universidade de São Paulo, 2008.

PEIXOTO, S. D. G.; ROCHA, F.; NISHIJIMA, M; Postali, F. A. S. Decentralization and corruption: evidence from primary health-care programmes. Applied Economics Letters, v. 19, p. 1885-1888, 2012.

PERSSON, T.; TABELLINI, G. Constitutional determinants of government spending. Mimeo. Bocconi University, 2000.

RODDEN, J. The dilemma of fiscal federalism: hard and soft budget constraints around the world. Mimeo. MIT, 2000.

SMITH, B. C. The decentralization of health care in developing countries: organizational options. Public Administration and Development, 17, p. 399-412, 1997.

STEGARESCU, D. Public sector decentralization: Measurement concepts and recent international trends. ZEW Discussion Paper, p. 04-74, 2004.

TANZI, V. On fiscal federalism: issues to worry about. Conference Notes, Conference on Fiscal Decentralization, IMF, Fiscal Affairs Department, Washington D.C., 2000.

TIEBOUT, C. A pure theory of local expenditures. Journal of Political Economy, 64, p. 416-424, 1956.

WORLD HEALTH ORGANIZATION. Global Health Observatory Data Repository. 2013. Disponível em: http://www.who.int/gho/database/en/. Acesso em 20/04/2017. 


\section{Sobre o autor}

Marislei Nishijima - marislei@usp.br

Instituto de Relações Internacionais, Universidade de São Paulo, São Paulo, SP.

Fernando Antonio Slaibe Postali - postali@usp.br

Departamento de Economia, Universidade de São Paulo, São Paulo, SP.

Fabiana Fontes Rocha - frocha@usp.br

Departamento de Economia, Universidade de São Paulo, São Paulo, SP.

Os autores agradecem o apoio financeiro do BNDES no âmbito do Programa de Fomento à Pesquisa em Desenvolvimento Econômico - PDE/BNDES/ANPEC. Agradecem também a Cássia Fernanda da Silva pela ajuda com os dados. O trabalho reflete somente a posição dos autores, devendo também todos os eventuais erros serem atribuídos somente a eles.

\section{Sobre o artigo}

Recebido em 11 de maio de 2015. Aprovado em 23 de dezembro de 2015. 